\title{
Adaptación de variedades de vid resistentes HPDs y cruces interespecíficos en condiciones tropicales como estrategia de paz en Colombia
}

\section{Adaptation of resistant grapevines varieties under tropical conditions: A social strategy for the post-conflict in Colombia}

\author{
J.E. Franco \\ Vinos de Colombia, Ibagué, Tolima, Colombia
}

\begin{abstract}
Resumen. En Colombia, la vid se puede considerar como un cultivo promisorio en la era del postconflicto. En esta investigación se evaluó la adaptación de más de cincuenta variedades de vid resistentes a las enfermedades, híbridos productores directos (HPDs) y cruces interespecíficos bajo condiciones tropicales, con el enfoque de encontrar variedades con resistencia para soportar los ataques de Plasmopara viticola, Guignardia bidwelii, Phomopsis viticola, Botrytis cinerea, Uncinula necator, entre otras; así como sus cualidades productivas y de calidad. Otra meta es reducir el número de aplicaciones de agroquimicos por periodo productivo y así lograr que la vid sea un cultivo más amigable con el medio ambiente y económicamente más rentable. Nuestra investigación se ha desarrollado en áreas del valle interandino en el centro de Colombia (departamento del Tolima) en el bosque seco tropical. Esta investigación tiene como propósito, constituirse en una herramienta de implementación de cultivo de la vid, viable económica, social y ambientalmente, como medio de subsistencia a familias campesinas, desplazados y desmovilizados en la era del postconflicto colombiano. A su vez, representa una alternativa de reemplazo a los cultivos ilícitos como la coca y la marihuana, que han prosperado en zonas afectadas por la guerra en Colombia.
\end{abstract}

\begin{abstract}
In Colombia, the grapevine can be considered as a promising crop over the post-conflict era. This research evaluated the local adaptation of more than fifty vine varieties resistant to diseases, direct producer hybrids (DPHs) and interspecific test crosses under tropical conditions with the approach of finding varieties with enough resistance to withstand the attacks of Plasmopara viticola, Guignardia bidwelii, Phomopsis viticola, Botrytis cinerea, Uncinula necator, among others; as well as its productive and quality characteristics. Another goal of our research is to reduce the number of applications of agrochemicals per productive period and thus doing the vine a more environment-friendly and economically more profitable crop. Our research has been developed in areas of the inter-Andean valley in central Colombia (department of Tolima) in the tropical dry forest life zone. This research is projected to become an implementation tool for the cultivation of the vine, economically, socially and environmentally viable; as a means of subsistence for peasant families, displaced and demobilized people in the post-conflict era of Colombia. In turn, it represents a replacement alternative to illicit crops such as coca and marijuana, which have flourished in areas affected by the war in Colombia.
\end{abstract}

\section{Antecedentes}

El cultivo de la vid en ubicaciones tropicales $23^{\circ} 26^{\prime} 14^{\prime \prime} \mathrm{N}$ y $23^{\circ} 26^{\prime} 14 \mathrm{~S}$, se ha convertido en una importante fuente de ingresos a países en vías de desarrollo y en una fuente local de vinos y fruta fresca para consumo [1].

En Colombia el cultivo de la vid se ha relegado a uvas de mesa; de las variedades Italia, Red Globe, Alfonso Lavallé, Isabella y algunas otras minoritarias [2]. En la producción de uvas para vinos nosotros hemos venido trabajando con la investigación e incorporación al inventario nacional variedades clásicas europeas, Malbec, Syrah, Tempranillo, Marselan, Cabernet Sauvignon, Petit Verdot, Macabeo y otras tantas completando una colección de más de 150 variedades en su mayoría Vitis vinífera.
En Colombia existen amplias zonas agrícolas donde normalmente se producen dos cosechas de uva al año, pero las lluvias suelen superar los $600 \mathrm{~mm}$ por cosecha lo que obliga a repetidas fumigaciones con agroquímicos y esto se agrava cuando el fenómeno meteorológico de "La Niña", duplica la cantidad de lluvia esperada.

La Vitis vinífera tiene una resistencia casi nula a los hongos como el Plasmopara vitícola, Guignardia bidwelii, Phomopsis vitícola, Botrytis cinérea, Uncinula necátor, lo que obliga a aplicaciones frecuentes las cuales muestran una agresividad ambiental muy alta, así como producen riesgos de residualidad de agroquímicos en el producto final, ya sea Vino, Jugo o Uvas para consumo en fresco.

Existe una gran necesidad de producción local de uvas para la elaboración de vinos y uvas industriales, así como 


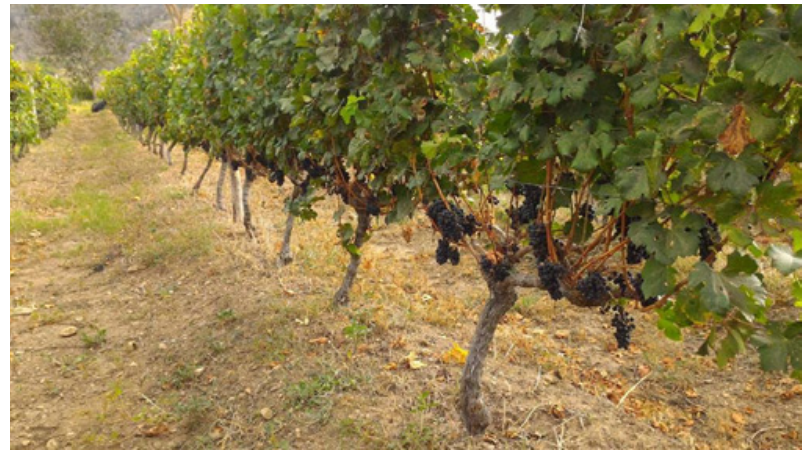

Figura 1. Marselan antes de cosecha.

de consumo en fresco; existen amplias áreas agrícolas en zonas que fueron afectadas por la guerra y que gracias a sus condiciones climáticas el cultivo de uva es una gran opción dado que consume una gran cantidad de mano de obra, que puede ser cubierta por desplazados, excombatientes desmovilizados y campesinos que quieren restituir cultivos ilícitos.

\section{Objetivo}

Esta investigación tiene como objetivo medir la capacidad de adaptación y resistencia a las enfermedades de variedades de cruces interespecíficos y HPDs a las condiciones tropicales; con la finalidad de introducir nuevas variedades al mercado que minimicen el uso de agroquímicos.

Esta investigación contempla los siguientes parámetros de adaptación:

- Agronómica

- Analiza la capacidad de crecimiento y vigor de la planta en condiciones tropicales y su capacidad de brotación.

- Resistencia

- Se exponen las vides a diversas enfermedades típicas de la vid y se analiza su impacto en el desarrollo de la planta.

- Productividad

- Se analiza la capacidad productiva de las vides.

- Calidad

- Se evalúa la calidad de la uva bajo cada uno de los parámetros establecidos para Uva de Vino y Uva de mesa.

\section{Protocolo}

Se importaron vides de viveros certificados desde el estado de New York en USA, se importaron 6 plantas por variedad, estas fueron sembradas en espaldera con poda royat doble brazo a una distancia entre plantas de $1.2 \mathrm{mt}$. y $2.5 \mathrm{mt}$. entre filas.

Las plantas fueron desarrolladas durante dos años durante los cuales se evaluaron la resistencia a las enfermedades fúngicas, capacidad de brotación, capacidad de producción y calidad; en el caso de las uvas de vino, su calidad de vino final y en el caso de las uvas de mesa, su sabor, resistencia al transporte y a la refrigeración.

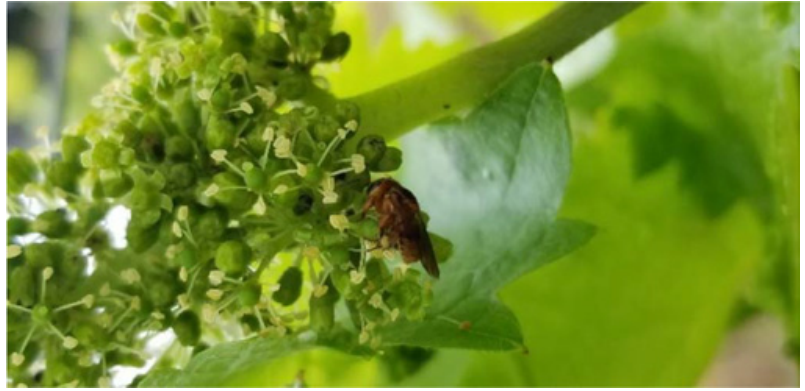

Figura 2. Abejas nativas alimentandose de las flores de Chardonnel.

\section{Resultados}

Hemos evaluado las siguientes variedades:

Alden, Baco, Caco, Cayuga, Chamburcin, Chardonel, Chelois, Colobel, Concord, Concord Seedless, Einset Seedless, Espirit, Frontenac, Glenora, Golden Muscat, Higgins, King of North, La crosse, Leon Millot, Lorelei, Niagara, Norton, New York Muscat, Oberlin Noir, Ontario, Regent, Reliance Seedless, Ruby Red, Seyval, Somerset Seedless, Soverreing Coronation Seedless, St. Vincent, Thomcord Seedless, Traminette, Vanessa, Venus, Victoria [3].

De este listado encontramos que las siguientes variedades totalmente inadaptadas que no pudieron sobrevivir como es el caso de Alden, Esprit, Higgins, Frontenac, Lorelei, Soverreing Coronation Seedless y Victoria.

Encontramos que la mayoría de estas variedades son de zonas muy norteñas, por lo que parece que la falta de frio estacional no les permitió sobrevivir.

Dentro de las variedades sobrevivientes han mostrado resultados variables, siendo una constante algunos problemas de brotación, pero hay algunas que pudieron adaptarse y producir de una manera viable y ambientalmente amigable; dentro de las mejor adaptadas fueron:

Baco, Cayuga, Chardonel, Chelois, Colobel, Glenora, Golden Muscat, Niagara, Regent, Seyval y Traminette.

De estas plantas se sembraron a pie franco 50 plantas por variedad; las variedades con aptitud para elaborar vinos fueron elaboradas de la siguiente forma las tintas sin roble, en tanques de acero, levaduras seleccionadas con temperatura controlada de $28^{\circ} \mathrm{C}$ y maceración de 18 días; las blancas prensado, enzimas pectolíticas, fermentado en acero con levaduras seleccionadas a $18^{\circ} \mathrm{C}$ y estabilización tartárica.

Los resultados generales fueron los siguientes:

Baco, Variedad negra, vigorosa y muy resistente a las enfermedades e insectos, produce en gran cantidad con calidad y color, resalta su carácter especiado; su calificación en vino en cata fue de 7.9.

Cayuga, Variedad blanca, vigor medio, libre de enfermedades, produce en abundancia, da vinos ácidos refrescantes aptos para la elaboración de vinos espumosos y blancos jóvenes; su calificación en vino en cata fue de 8.3.

Chardonel, Variedad blanca, vigorosa, libre de enfermedades e insectos, producción media, pero de 
alta calidad, equiparable a su parental genético el Chardonnay, su calificación en vino en cata fue de 8.7 [4].

Chelois, Variedad negra, vigorosa y muy resistente a las enfermedades, buena producción y calidad; su calificación en vino en cata fue de 8.5.

Colobel, Variedad negra, resistente, vigorosa de cascara gruesa, es una variedad tintorera por lo que sus niveles de color son muy altos, buena producción y buena calidad; su calificación en vino en cata fue de 8.5.

Golden Muscat, Variedad blanca, de gran tamaño, resistencia a las enfermedades media, alta producción, exquisito sabor a moscatel con dejos de lambrusca, podría ser una excelente uva de mesa, pero su problema de desgrane la limita su uso a elaboración de vinos y usos industriales; su calidad de vino es media alta, siendo su calificación media de 7.7.

Regent, Variedad tinta, resistencia media a las enfermedades; ligeros problemas de brotación por lo que se debe usar podas cortas; alta producción, alta calidad y color, taninos potentes, su calidad sorprendió obteniendo una de las mayores calificaciones en cata 9.1 .

Seyval, Variedad blanca, vigorosa, productiva e inmune a las enfermedades fúngicas y muy precoz, su debilidad es la susceptibilidad a la botrytis; los vinos son frescos de buena calidad su calificación en cata fue de 8.3.

Traminette, Variedad blanca de alta calidad, buena resistencia a las enfermedades, producción media muy aromática, resalta su parental genético Gewürztraminer del cual hereda su aroma característico; su calidad fue alta y su calificación en cata llegó a 8.8 [5].

De las variedades de mesa evaluadas Las de mejores resultados fueron las siguientes:

Glenora, Uvas rosada con semilla de buen tamaño productiva y resistente de buen sabor afrutado, cascara gruesa excelente resistencia al transporte y refrigeración.

Niagara, Uva blanca con semilla de tamaño medio resistente a las enfermedades muy productiva y buen sabor, resistencia media al transporte.

Cabe resaltar que actualmente seguimos evaluando variedades y hay algunas que con manejo técnico y mejor elección de zona climática han mejorado ostensiblemente y se perfilan viables para cultivo.

\section{Estrategia de paz}

Nuestro centro de investigación está ubicado en Colombia en el departamento de Tolima, departamento que ha sufrido desde hace muchos años la violencia de los grupos armados como las FARC, que vieron su nacimiento a $100 \mathrm{~km}$ de donde tenemos nuestro centro de investigación; por eso nuestro proyecto siempre fue visto como una estrategia de paz dado que permite que los desmovilizados del proceso de paz puedan cultivar uva y ser vendidas a las empresas productoras de vinos y al mercado local de uvas de mesa.
Posterior a la evaluación de las variedades que fueron viables para cultivo, se les entregará diez plantas a campesinos de la zona afectada por la violencia, recibieran capacitación de cultivo de la vid; este contacto inicial tiene como fin que los campesinos conozcan la vid y se enamoren de ella.

La siguiente fase fue con los campesinos que mostraron interés, se les facilitará 50 plantas para que iniciaran un cultivo comercial.

El proyecto se encuentra en desarrollo; pero la aceptación ha sido bastante buena y se considerará ampliar este plan a otras zonas afectadas si logramos conseguir apoyo a nuestro proyecto "Uvas para la Paz".

\section{Conclusiones}

El cultivo de variedades resistentes ha abierto un panorama enorme a la viticultura en Colombia, dado que permite cultivar uvas en zonas que antes era imposible por la cantidad de lluvias lo cual hace inviable el cultivo de Vitis vinífera.

Estas variedades al haberse minimizado las aplicaciones de agroquímicos han resultado ser un sitio de alimentación de las abejas nativas.

Dado que la mayoría de estas vides tienen genética americana tienen resistencia inherente a la filoxera y nematodos; por lo que se pueden reproducir por estacas, lo cual baja el costo de implementación.

La minimización de las aplicaciones de fungicidas ha sido notable, pasamos de doce aplicaciones en Vitis vinífera a cuatro en las de resistencia media y dos o ninguna en las variedades inmunes; lo que hace que el cultivo de la vid sea viable para familias campesinas de bajos recursos.

Los dos factores de bajos costos de implantación, cultivo y mantenimiento; esto aunado a las dos cosechas al año, se ha planteado como una alternativa de cultivo en zonas de postconflicto y como una alternativa a los cultivos ilícitos como la coca y la marihuana.

\section{Referencias}

[1] J.V. Possingham, P.R. Clingeleffer, G.H. Kerridge, Breeding Grapes for Tropical Enviroments, (Australia, CSIRO Division of horticulture, 1986)

[2] Ministerio de agricultura de Colombia, https:// www.minagricultura.gov.co/noticias/ Paginas/La-uva-sin-semilla-germina.aspx, (24/04/2015)

[3] A. Morata, I. Loira, Grape and Wine Biotechnol. 405 (2016)

[4] B.I. Reisch, R.M. Pool, W.B. Robinson, T. Henick-Kling, J.P. Watson, K.H. Kimball, M.H. Martens, G.S. Howell, D.P. Miller, C.E. Edson, J.R. Morris, New York's Food and Life Sciences Bulletin 'CHARDONEL' Grape (1990)

[5] B.I. Reisch, R.M. Pool, W.B. Robinson, T. Henick-Kling, B.K. Gavitt, J.P. Watson, M.H. Martens, R.S. Luce, H.C. Barrett, N.Y. Food and Life Sci. Bull. 149 (1996) 